\title{
O papel do enfermeiro no manejo da dor nos pacientes em cuidados paliativos oncológicos: uma revisão integrativa
}

O objetivo é identificar o papel do enfermeiro no controle efetivo da dor oncológica. O estudo foi do tipo revisão integrativa realizado entre abril e maio de 2021 buscando responder a questão norteadora: 'Qual é o papel do enfermeiro no manejo da dor oncológica?'. No qual, 7 artigos atendiam o critério de elegibilidade todos no idioma português. Os artigos selecionados foram publicados na Literatura Latino-americana e do Caribe em Ciências da Saúde (LILACS), na Base de Dados de Enfermagem (BDENF) e no Sistema Online de Busca e Análise de Literatura Médica (MEDLINE). A avaliação imprecisa dos quadros álgicos, a utilização insuficiente de medicações analgésicas disponíveis, o preconceito para com o uso de medicamentos opióides e a falta de conhecimento com relação ao manejo da dor na equipe de saúde implicam no controle inadequado da dor. A dor decorrente do câncer, independente de sua intensidade, é um fator que implica na recuperação do paciente. Com os cuidados especializados, além de terapias complementares, houve melhoras na dor. 0 enfermeiro assume papel fundamental na mensuração da dor do paciente oncológico e a mensuração desses sintomas é de grande importância para descobrir qual a prevalência dos mesmos e quais manejos estão tendo seus resultados esperados, sendo assim, atuando diretamente na qualidade de vida do paciente.

\section{The role of the nurses in pain management in patients in palliative oncological care: an integrative review}

The objective is to identify the role of nurses in effective cancer pain control. An integrative review study, carried out between April and May 2021, seeking to answer the guiding question: 'What is the role of nurses in cancer pain management?'. In which, 7 articles met the eligibility criteria, all in Portuguese. The selected articles were published in the Latin American and Caribbean Literature on Health Sciences (LILACS), in the Nursing Database (BDENF) and in the Online System for Search and Analysis of Medical Literature (MEDLINE). Inaccurate evaluation of pain conditions, insufficient use of available analgesic medications, prejudice towards the use of opioid medications and lack of knowledge regarding pain management in the healthcare team imply inadequate pain control. Pain resulting from cancer, regardless of its intensity, is a factor that affects the patient's recovery. With specialized care, in addition to complementary therapies, there were improvements in pain. The nurse assumes a fundamental role in measuring the pain of cancer patients and the measurement of these symptoms is of great importance to find out what their prevalence is and what managements are having their expected results, thus, acting directly on the quality of life of the patient.

Keywords: Cancer pain; Nursing; Pain Management.

Topic: Enfermagem Geral

Reviewed anonymously in the process of blind peer.

Ana Luiza Rosa Manoel (iD

Universidade de Sorocaba, Brasil

http://lattes.cnpq.br/3532644968982555

http://orcid.org/0000-0002-0541-8671

analuizarsm96@gmail.com

Victória Sofia Moreira Marques Penteado (D)

Universidade de Sorocaba, Brasil

http://lattes.cnpq.br/5444418254091968

http://orcid.org/0000-0001-5297-1261

vicmaques@gmail.com

Lucas Bueno de Oliveira (D)

Universidade de Sorocaba, Brasil

http://lattes.cnpq.br/6017620994952226

http://orcid.org/0000-0001-5446-3471

lbuenodeoliveira@hotmail.com
Received: 12/06/2021

Approved: 09/07/2021
Débora Cabral Nunes Polaz (iD)

Universidade de Sorocaba, Brasil

http://lattes.cnpq.br/9342411050092970

http://orcid.org/0000-0003-4800-672X

debora.polaz@gmail.com

Leandro Aparecido Souza

Universidade de Sorocaba, Brasil

http://lattes.cnpq.br/6090315154831086

http://orcid.org/0000-0001-8828-9918

eandroapsouza14@gmail.com
Referencing this:

MANOEL, A. L. R.; PENTEADO, V. S. M. M.; OLIVEIRA, L. B.; POLAZ, D. C. N.; SOUZA, L. A.. O papel do enfermeiro no manejo da dor nos pacientes em cuidados paliativos oncológicos: uma revisão integrativa. Scire Salutis, v.11, n.3, p.20-27, 2021. DOI:

http://doi.org/10.6008/CBPC2236-9600.2021.003.0004 


\section{INTRODUÇÃO}

Estima-se que, em 2020, a incidência de casos novos de neoplasias em homens e mulheres foi de 309.750 e 316.280 , respectivamente, e em 2019 , a mortalidade por neoplasias em homens e mulheres foi de 121.686 e 110.344, respectivamente, segundo o Instituto Nacional de Câncer (INCA). Conforme observamos o aumento da quantidade de casos novos, a necessidade de termos profissionais de saúde preparados para aplicação de cuidados paliativos em pacientes e em suas famílias cresce paralelamente a estes números (INCA, 2021).

O crescimento desordenado das células e a possibilidade da invasão dessas células em outras regiões do corpo são o que caracteriza a fisiopatologia do câncer e suas metástases. Dentro de sua normalidade, o mecanismo de controle do crescimento celular é regulado por fatores estimulantes e inibidores, de forma equilibrada, havendo crescimento celular sem ativação do mecanismo através de estímulos variantes. Quando há a ruptura desse mecanismo de controle da multiplicação celular e o crescimento e divisão desordenada de uma célula, sem que seja uma necessidade do tecido, sua resultante é uma neoplasia ou tumor, podendo este ser benigno ou maligno (INCA, 2015).

Dentre as características sintomáticas dessa doença e de seu tratamento, a dor é uma das mais presentes. Quando oncológica, essa dor pode ser nociceptiva e/ou neuropática, sendo mais frequente a nociceptiva na dor musculoesquelética, que tem um importante papel na predição de mortalidade (ANCP, 2012). A dor afeta significativamente a qualidade de vida do paciente, estimando-se que esteja presente em cerca de $30 \%$ dos diagnosticados e em $80 \%$ dos que estão em fase terminal. Esse é um dos motivos que a partir do ano de 2000 a dor é considerada o quinto sinal vital, na qual é mensurada através de diversas escalas para aprimorar a assistência (KLAUMANN et al., 2008).

De acordo com Klaumann et al. (2008) a dor fisiológica é a que induz respostas protetoras, como a reação de fuga, que é o sinal típico da dor aguda, produzida por estímulos intensos na superfície da pele. A dor visceral e a dor somática profunda são causadas por estímulos inevitáveis. Subdividida em nociceptiva e neuropática, está a dor persistente. Onde respectivamente a nociceptiva é resultado da ativação de nociceptores da pele e outros tecidos em resposta a uma lesão tecidual, acompanhada de inflamação e a neuropática ou neurogênica está relacionada a lesões de nervos periféricos ou do sistema nervoso central (KLAUMANN et al., 2008).

Em síntese, a dor aguda tipicamente surge do trauma de tecidos moles ou inflamação e está relacionada com um processo adaptativo biológico para facilitar o reparo tecidual e cicatricial. Enquanto a dor crônica é definida como duração maior que 3 a 6 meses. Pode manifestar-se espontaneamente ou ser provocada por vários estímulos externos. A dor crônica além de simplesmente manifestar-se por um longo período de tempo, implica numa síndrome debilitante que interfere diretamente na qualidade de vida do paciente e é mais difícil de controlar com métodos de analgesia convencional (KLAUMANN et al., 2008).

Ao abordar a atuação do enfermeiro nessa área de uma forma ampla, foi dado enfoque para encontrar informações que trazem a importância do controle efetivo da dor oncológica, tendo como base 
terapêutica medicamentos, intervenções terapêuticas não medicamentosas e a busca pelo bem-estar funcional e emocional do paciente e sua família. Diante do contexto apresentado, qual é o papel do enfermeiro no manejo da dor oncológica?

Portanto o presente estudo tem por objetivo identificar o papel do enfermeiro no controle efetivo da dor oncológica, tendo como base terapêutica medicamentos, intervenções terapêuticas não medicamentosas e a busca pelo bem-estar funcional e emocional do paciente e sua família.

\section{METODOLOGIA}

Trata-se de uma revisão integrativa da literatura a partir de busca eletrônica por artigos científicos indexados nas bases de dados: Literatura Latino-americana e do Caribe em Ciências da Saúde (LILACS), Base de Dados de Enfermagem (BDENF) e o Sistema Online de Busca e Análise de Literatura Médica (MEDLINE), a partir dos descritores: Dor do câncer, Enfermagem e Manejo da Dor, sendo a busca realizada com o cruzamento destes descritores, utilizando o operador 'and'.

Para a consolidação dos estudos, seguiram-se as seguintes etapas: escolha do tema, levantamento bibliográfico preliminar, formulação do problema, busca de fontes, leitura do resumo, e os que foram considerados nesta pesquisa, a leitura foi na íntegra, organização lógica do assunto, e a redação do texto. Utilizou-se como critérios de inclusão artigos originais disponíveis na íntegra, que responderam aos objetivos do estudo, publicados entre o período de 2016 a 2021 no idioma português e como critérios de exclusão: textos em formato de resumo, língua estrangeira, publicações anteriores de 2016, bem como teses e dissertações e artigos que não atendiam aos objetivos do estudo.

A busca resultou em 486 artigos, dos quais, após análise, foram descartados 479 que não atendiam aos critérios de inclusão, sendo selecionados 7 artigos, apresentados no Fluxograma 1 como síntese das principais informações obtidas.

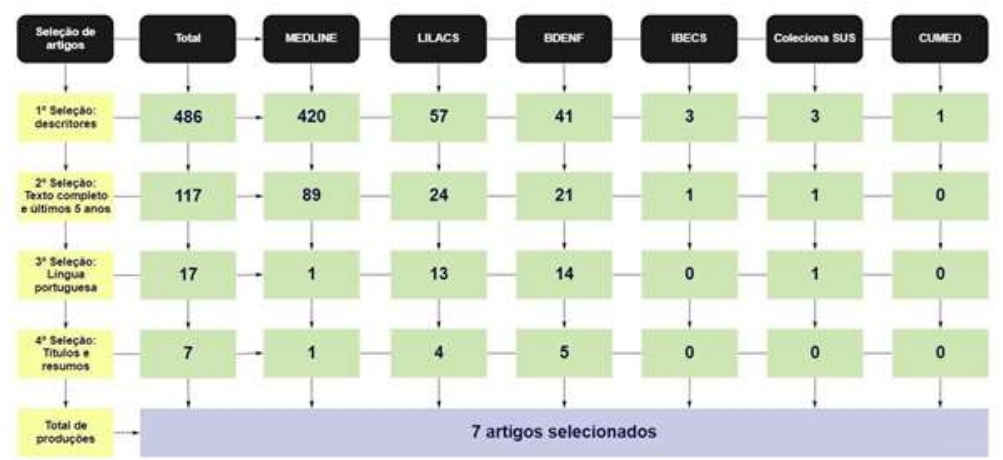

Fluxograma 1: Mostra como se deu a busca em cada base de dados

A coleta dos dados ocorreu entre março e abril de 2021 e após a seleção dos trabalhos procedeu-se às leituras exploratórias, seletiva, analítica e por fim a redação do trabalho. Foram analisadas cinco categorias dos artigos: Título, ano de publicação, autores, objetivos e conclusão do estudo da literatura, dos quais os artigos que constituíram o estudo abordaram a o papel do enfermeiro no controle efetivo da dor oncológica, tendo como base terapêutica medicamentos, intervenções terapêuticas não medicamentosas e a busca pelo bem-estar funcional e emocional do paciente e sua família. 


\section{RESULTADOS E DISCUSSÃO}

\section{Os 7 artigos selecionados e analisados neste estudo estão apresentados no quadro a seguir}

Quadro 1: Caracterização da produção científica analisada segundo título do estudo, anos de publicação, autores, objetivo e resultados, 2021

\begin{tabular}{|c|c|c|c|c|c|}
\hline $\mathbf{N}^{\circ}$ & $\begin{array}{c}\text { Título } \\
\text { do estudo }\end{array}$ & $\begin{array}{c}\text { Ano de } \\
\text { Publicação }\end{array}$ & Autores & Objetivo & Resultados \\
\hline A01 & $\begin{array}{l}\text { Conhecimento de } \\
\text { enfermeiros } \\
\text { residentes sobre o } \\
\text { manejo da dor } \\
\text { oncológica: estudo } \\
\text { transversal }\end{array}$ & 2016 & $\begin{array}{l}\text { Ferreira } \\
\text { et al. }\end{array}$ & $\begin{array}{l}\text { Avaliar o conhecimento de } \\
\text { enfermeiros residentes sobre o } \\
\text { manejo da dor no câncer e as } \\
\text { variáveis sociodemográficas e } \\
\text { profissionais associadas. }\end{array}$ & $\begin{array}{l}\text { O artigo indicou que a maioria dos enfermeiros } \\
\text { residentes avaliados apresentava } \\
\text { conhecimento inapropriado em relação ao } \\
\text { manejo da dor no câncer. O estudo também } \\
\text { apontou que não houve diferença no nível de } \\
\text { conhecimento entre os residentes do primeiro } \\
\text { e segundo ano, e o conhecimento foi } \\
\text { dependente do tempo da formação. } \\
\text { Os enfermeiros residentes participantes do } \\
\text { estudo demonstraram moderado } \\
\text { conhecimento prévio sobre os aspectos do } \\
\text { manejo da dor, porém, quanto aos efeitos de } \\
\text { intervenção educativa sobre o conhecimento e } \\
\text { as atitudes frente à dor foram considerados } \\
\text { insatisfatórios. }\end{array}$ \\
\hline $\mathrm{A02}$ & $\begin{array}{l}\text { Fatores que interferem } \\
\text { no gerenciamento do } \\
\text { cuidado ao paciente } \\
\text { oncológico em um } \\
\text { hospital geral }\end{array}$ & 2016 & $\begin{array}{l}\text { Peiter } \\
\text { et al. }\end{array}$ & $\begin{array}{l}\text { Indicar os fatores que interferem } \\
\text { no gerenciamento do cuidado de } \\
\text { enfermagem frente aos pacientes } \\
\text { oncológicos internados em um } \\
\text { hospital geral localizado no sul do } \\
\text { país. }\end{array}$ & $\begin{array}{l}\text { Os fatores interferentes identificados, foram: o } \\
\text { gerenciamento da unidade realizado pelo } \\
\text { enfermeiro, a necessidade de capacitações, o } \\
\text { vínculo com a família, o relacionamento } \\
\text { interpessoal na equipe, a não-especificidade da } \\
\text { clínica, o processo de morte e a dor oncológica, } \\
\text { sendo esta muitas vezes negligenciada pelo } \\
\text { profissional por não conseguir suprimi-la com } \\
\text { analgésicos. A avaliação imprecisa dos quadros } \\
\text { álgicos, a utilização insuficiente de medicações } \\
\text { analgésicas disponíveis, o preconceito para } \\
\text { com o uso de medicamentos opióides e a falta } \\
\text { de conhecimento com relação ao manejo da } \\
\text { dor na equipe de saúde implicam no controle } \\
\text { inadequado da dor. }\end{array}$ \\
\hline A03 & $\begin{array}{l}\text { Produção científica de } \\
\text { enfermeiros brasileiros } \\
\text { sobre enfermagem e } \\
\text { oncologia: revisão } \\
\text { narrativa da literatura }\end{array}$ & 2019 & $\begin{array}{l}\text { Rolim et } \\
\text { al. }\end{array}$ & $\begin{array}{l}\text { Conhecer o que tem sido } \\
\text { produzido por enfermeiros } \\
\text { brasileiros sobre enfermagem e } \\
\text { oncologia. }\end{array}$ & $\begin{array}{l}\text { A dor decorrente do câncer pode ser tanto } \\
\text { aguda crônica e independente de sua } \\
\text { intensidade é um fator que implica na } \\
\text { recuperação do paciente. A equipe de } \\
\text { enfermagem deve estar atenta às queixas } \\
\text { subjetivas (relatos verbais, expressão facial e } \\
\text { pelo olhar) e mensurar a dor do paciente, para } \\
\text { estabelecer um plano de cuidado adequado e } \\
\text { individualizado. O controle da dor é } \\
\text { considerado indicador de qualidade de vida e } \\
\text { de assistência, e por isso é necessária a } \\
\text { implementação de estratégias para o seu } \\
\text { manejo no paciente oncológico, sejam elas } \\
\text { farmacológicas ou não, incluindo terapias } \\
\text { complementares. }\end{array}$ \\
\hline A04 & $\begin{array}{l}\text { Implementação da } \\
\text { avaliação da dor como o } \\
\text { quinto sinal vital }\end{array}$ & 2018 & $\begin{array}{l}\text { Castro } \\
\text { et al. }\end{array}$ & $\begin{array}{l}\text { Analisar a implementação da } \\
\text { avaliação da dor como o quinto } \\
\text { sinal vital. }\end{array}$ & $\begin{array}{l}\text { Aumento da qualidade da assistência após a } \\
\text { implementação da avaliação e registo da dor. } \\
\text { Auxílio na frequência de administração dos } \\
\text { analgésicos após mensuração da dor. }\end{array}$ \\
\hline A05 & $\begin{array}{l}\text { Acompanhamento } \\
\text { longitudinal do manejo } \\
\text { de sintomas em serviço } \\
\text { especializado de } \\
\text { cuidados paliativos } \\
\text { oncológicos (5) }\end{array}$ & 2020 & $\begin{array}{l}\text { Reis et } \\
\text { al. }\end{array}$ & $\begin{array}{l}\text { Analisar a funcionalidade e os } \\
\text { sintomas de pacientes } \\
\text { oncológicos em fim de vida } \\
\text { durante internação em unidade } \\
\text { especializada em cuidados } \\
\text { paliativos. }\end{array}$ & $\begin{array}{l}\text { Em unidade especializada, houve melhora dos } \\
\text { sintomas dor, cansaço e tristeza, como também } \\
\text { da funcionalidade dos participantes em duas } \\
\text { semanas de internação. }\end{array}$ \\
\hline
\end{tabular}




\begin{tabular}{|c|c|c|c|c|c|}
\hline A06 & $\begin{array}{l}\text { Avaliação de sintomas } \\
\text { em pacientes } \\
\text { oncológicos internados } \\
\text { em unidade de cuidados } \\
\text { paliativos exclusivos (6) }\end{array}$ & 2020 & $\begin{array}{l}\text { Neves } \\
\text { et al. }\end{array}$ & $\begin{array}{l}\text { Avaliar os sintomas em pacientes } \\
\text { oncológicos internados em uma } \\
\text { unidade de cuidados paliativos } \\
\text { exclusivos. }\end{array}$ & $\begin{array}{l}\text { Houve melhor manejo da dor, colaborando } \\
\text { com a melhora da qualidade de vida dos } \\
\text { indivíduos; ocorreu, entretanto, aumento do } \\
\text { cansaço e sonolência. Também se mostra } \\
\text { relevante ao permitir a reflexão quanto aos } \\
\text { sintomas que mais reverberam no fim de vida, } \\
\text { empoeirando o enfermeiro no cuidado junto } \\
\text { com a equipe interdisciplinar, além de ratificar } \\
\text { a importância dos cuidados paliativos. }\end{array}$ \\
\hline A07 & $\begin{array}{l}\text { Resultados de } \\
\text { enfermagem para } \\
\text { avaliação da dor de } \\
\text { pacientes em cuidado } \\
\text { paliativo (7) }\end{array}$ & 2018 & $\begin{array}{l}\text { Mello et } \\
\text { al. }\end{array}$ & $\begin{array}{l}\text { Selecionar resultados e } \\
\text { indicadores da Nursing Outcomes } \\
\text { Classification (NOC) para avaliar } \\
\text { pacientes oncológicos em } \\
\text { cuidados paliativos com os } \\
\text { diagnósticos de enfermagem de } \\
\text { Dor Aguda e Crônica; construir as } \\
\text { definições conceituais e } \\
\text { operacionais dos indicadores. }\end{array}$ & $\begin{array}{l}\text { Foram selecionados resultados da NOC pelos } \\
\text { especialistas, permitindo a elaboração de um } \\
\text { instrumento composto de oito resultados e } \\
\text { dezenove indicadores com definições } \\
\text { conceituais e operacionais para avaliar os Des } \\
\text { Dor Aguda e Dor Crônica na assistência de } \\
\text { enfermagem ao paciente em cuidados } \\
\text { paliativos. }\end{array}$ \\
\hline
\end{tabular}

Ferreira et al. 2016 expressa que o manejo da dor na equipe multidisciplinar cria um papel de destaque para o enfermeiro, que além de aplicar o tratamento medicamentoso antiálgico, também é responsável pela avaliação da dor, da resposta às terapêuticas aplicadas e de possíveis reações adversas, sendo estes fatores importantes para traçar o caminho do tratamento específico para este paciente. Já Peiter et al. (2016), declara que o papel do enfermeiro frente ao paciente oncológico deve, também, ser educativo, esclarecendo dúvidas sobre o diagnóstico e orientando-o quanto ao tratamento. Neves et al. (2020), indica que a atividade da equipe de enfermagem também é essencial para o manejo de necessidades psicoespirituais.

Castro et al. (2018) abordou a importância da avaliação da dor para melhorar a assistência ao paciente. Em relação a equipe de enfermagem selecionada para participar da pesquisa, $75 \%$ responderam que o instrumento para a mensuração da dor influenciou positivamente na elaboração do diagnóstico de enfermagem e $25 \%$ consideraram que pouco influenciou, porém, no total, não houve queixas de que o registro da dor não contribuiu na qualidade da assistência de enfermagem.

Rolim et al. (2019), aponta que a dor decorrente do câncer pode ser aguda ou crônica, independente da sua intensidade e com base nessas informações, conclui-se que é importante que o enfermeiro conheça as particularidades da patologia e principalmente estar atento às queixas subjetivas, bem como mensurar a dor do paciente para que a sistematização da assistência de enfermagem seja elaborada de forma individualizada e adequada.

De acordo com o estudo realizado por Reis et al. (2020) com indivíduos no final de vida, internados em uma unidade especializada em cuidados paliativos, que tiveram seus sintomas mensurados através da Escala de Performance Paliativa (PPS) e avaliação utilizando a Escala de Avaliação de Sintomas de Edmonton (ESAS-r), que avaliam respectivamente: dor, cansaço, sonolência, náusea, apetite, falta de ar, depressão, ansiedade e bem-estar, os benefícios dos cuidados foram observados em decorrência da melhora dos sintomas de dor, cansaço, tristeza e funcionalidade. Porém, requerem maiores pesquisas sobre as estratégias efetivas direcionadas a melhora do cansaço, já que esse sintoma tem limitação do tratamento e desconhecimento dos mecanismos fisiopatológicos.

Em outro estudo quantitativo prospectivo relacionado a avaliação de sintomas em pacientes 
oncológicos de uma clínica de cuidados paliativos, Neves et al. (2020), após admissão desses pacientes e utilização das mesmas escalas, ESAS-r e PPS, houve melhor manejo da dor, proporcionando mais qualidade de vida. Porém, ocorreu aumento do cansaço e sonolência, abordando a reflexão relacionada aos sintomas que mais reverberam no fim de vida.

Castro et al. (2018) retrata a implementação da dor como quinto sinal vital, visto que a dor é subjetiva e somente o paciente pode descrevê-la de forma detalhada. Observou-se neste estudo uma falha na coleta de dados, devido a não utilização dos critérios de avaliação da dor pela equipe de enfermagem selecionada para o estudo. Este fato impossibilitou melhores resultados, porém, com os dados obtidos foi possível analisar que a mensuração da dor auxiliou na frequência de administração dos analgésicos.

A pesquisa realizada por Ferreira et al. (2016) acusa que cerca de $68,2 \%$ dos enfermeiros residentes do hospital analisado em questão não têm conhecimento adequado sobre o manejo da dor oncológica. 0 autor argumenta que esse número se deu por conta das graduações não terem disciplinas voltadas à dor em específico, somente a abordado de modo esporádico entre os conteúdos de outras disciplinas da grade curricular. Essa falta de conhecimento adequado dos enfermeiros faz com que a crença em mitos sobre a manutenção da dor do câncer seja mais presente entre estes profissionais, gerando, assim, subestimação da queixa álgica dos pacientes, utilização de tratamentos analgésicos insuficientes e, ainda, fortalecimento dos mitos e das concepções errôneas dos pacientes e seus familiares acerca do tratamento.

Deste modo, Peiter et al. (2016) complementa a fala de Ferreira et al. (2016) apontando que esse conhecimento falho sobre o manejo da dor oncológica tem a necessidade de ampliação para que o planejamento assistencial da pessoa com câncer seja condizente a sua carência.

Sendo reflexo da pobreza do conhecimento técnico-científico sobre o manejo álgico oncológico, Peiter et al. (2016) e Ferreira et al. (2016) abordam o preconceito dos profissionais de saúde quanto a administração e prescrição de opióides. De acordo com Ferreira et al. (2016), esse medo se deve também pelas possíveis evoluções que estes medicamentos podem causar, sendo algumas delas: o vício e a depressão respiratória.

Uma das enfermeiras entrevistadas por Peiter et al. (2016) cita que tanto os profissionais de Enfermagem, quanto a equipe médica, acabam atribuindo a dor do paciente oncológico inteiramente ao câncer, justificando, portanto, o motivo desta estar presente numa grande proporção, o que acarreta um protelamento na prescrição de opióides e na aplicação desse tratamento. A equipe de saúde aguarda o aumento da dor para que as medidas medicamentosas sejam tomadas, levando a um sofrimento prolongado e desnecessário para o paciente com câncer.

Rolim et al. (2019) traz como opção para o tratamento da dor em pacientes oncológicos as terapias complementares, tais como: acupuntura, homeopatia, termalismo, terapia comportamental, plantas medicinais e fitoterapia. Relata que segundo pesquisas com pacientes submetidos a quimioterapia em uso de terapias complementares, estes apresentaram resultados positivos, alívio da dor, senso de autocontrole e conforto psicológico, redução de sinais e sintomas e até mesmo a regressão do câncer, 
além de uma maior proximidade com o profissional. Ainda neste estudo foi possível identificar a importância de outros métodos não farmacológicos para o alívio da dor decorrente do câncer. Estes são: aplicação de frio e/ou calor, massagem manual, relaxamento e distração dirigida.

Quanto ao profissional de Enfermagem frente à aplicação das Práticas Integrativas e Complementares (PICs), o Conselho Federal de Enfermagem COFEN resolve nos artigos $1^{\circ}$ e $2^{\circ}$ da Resolução Cofen - 197/1997 que estabelece e reconhece as Terapias Alternativas como qualificação do enfermeiro, visando que o mesmo 'deverá ter concluído e sido aprovado em curso reconhecido por instituição de ensino ou entidade congênere, com uma carga horária mínima de 360 horas (COFEN, 1997).

\section{CONCLUSÕES}

Conclui-se que o enfermeiro assume papel fundamental na mensuração da dor do paciente oncológico, visando queixas subjetivas, tais como relatos verbais, expressão facial e pelo olhar, além dos sinais fisiológicos que podem se manifestar como: a taquicardia, taquipneia, aumento da pressão arterial, palidez, sudorese e alterações das tensões musculares. A ansiedade e respostas emocionais também se mostram frequentes em situações de dor. A mensuração desses sintomas é de grande importância para descobrir qual a prevalência dos sintomas e quais manejos estão tendo seus resultados esperados.

Sabendo que o controle da dor é considerado um indicador de qualidade de vida, é importante implementar estratégias como as terapias complementares para o alívio da dor, não olhando somente para a administração de terapias medicamentosas. Levar em consideração a implementação da dor como quinto sinal vital é de grande avanço na qualidade do serviço prestado para pacientes em situação de dor decorrente do câncer, uma vez que esta prática permite melhor avaliação da dor e até mesmo redução da frequência de administração de analgésicos.

Sendo assim, por conta do enfermeiro exercer um papel que tem tanta importância na manutenção da qualidade de vida dos pacientes oncológicos, a insegurança relatada nos artigos analisados deve ser combatida com uma formação técnico-científica continuada, contendo um olhar profundo para o manejo da dor oncológica.

\section{REFERÊNCIAS}

COFEN. Conselho Federal de Enfermagem. Ações de enfermagem para o controle do câncer: fisiopatologia do câncer. COFEN, 2015.

CASTRO, C. C.; PEREIRA, A. K.; BASTOS, B. R.. Implementação da avaliação da dor como o quinto sinal vital. Revista de Enfermagem UFPE, p.3009-3014, 2018. DOI: https://doi.org/10.5205/1981-8963-v12i11a236994p3009$\underline{3014-2018}$

INCA. Instituto Nacional de Câncer. Causas e prevenção: estatísticas de câncer. INCA, 2021.

COFEN. Conselho Federal de Enfermagem. Resolução n. 197. Estabelece e reconhece as Terapias Alternativas como especialidade e/ou qualificação do profissional de Enfermagem. COFEN, 1997.
FERREIRA, F. S.; SANTOS, J.; MEIRA, K. C.. Conhecimento de enfermeiros residentes sobre o manejo da dor oncológica: estudo transversal. Online Brazilian Journal of Nursing, p. 694-703, 2016. DOI: http://dx.doi.org/10.1188/15.ONF.634$\underline{641}$

KLAUMANN, P. R.; WOUK, A. F. P. F.; SILLAS, T.. Patofisiologia da dor. Biblioteca Digital de Pediódicos, v.13, n.1, p.1-12, 2008. DOI: http://dx.doi.org/10.5380/avs.v13i1.11532

COFEN. Conselho Federal de Enfermagem. Manual de Cuidados Paliativos. COFEN, 2012.

MELLO, B. S.; ALMEIDA, M. A.; PRUINELLI, L.; LUCENA, A. F.. Resultados de enfermagem para avaliação da dor de pacientes em cuidado paliativo. Revista Brasileira de Enfermagem, p.70-78, 2021. DOI: https://doi.org/10.1590/0034-7167-2018-0307 
NEVES, K. E. S.; MUNIZ, T. S.; REIS, K. M. C.. Avaliação de sintomas em pacientes oncológicos internados em unidade de cuidados paliativos exclusivos. Revista Cogitare Enfermagem, v.25, 2020. DOI: http://dx.doi.org/10.5380/ce.v25i0.71660

PEITER, C. C.; CARMINHA, M. E. P.; LANZONI, G. M. M.; ERDMANN, A. L.. Fatores que interferem no gerenciamento do cuidado ao paciente oncológico em um hospital geral. Revista de Enfermagem da UFSM, p.404-413, 2016.
REIS, K. M. C.; JESUS, C. A. C.. Acompanhamento longitudinal do manejo de sintomas em serviço especializado de cuidados paliativos oncológicos. Revista Oficial Conselho Federal de Enfermagem, p.72-78, 2020.

ROLIM, D. S.; ARBOIT, E. L.; KAEFER, C. T.; MARISCO, N. S.; ELY, G. Z.; ARBOIT, J.. Produção Científica de Enfermeiros Brasileiros sobre Enfermagem e Oncologia: revisão Narrativa da Literatura. Ciências da Saúde UNIPAR, p.41-47, 2019.

A CBPC - Companhia Brasileira de Produção Científica (CNPJ: 11.221.422/0001-03) detém os direitos materiais desta publicação. Os direitos referem-se à publicação do trabalho em qualquer parte do mundo, incluindo os direitos às renovações, expansões e disseminações da contribuição, bem como outros direitos subsidiários. Todos os trabalhos publicados eletronicamente poderão posteriormente ser publicados em coletâneas impressas sob coordenação da Sustenere Publishing, da Companhia Brasileira de Produção Científica e seus parceiros autorizados. Os (as) autores (as) preservam os direitos autorais, mas não têm permissão para a publicação da contribuição em outro meio, impresso ou digital, em português ou em tradução. 